Ergod. Th. \& Dynam. Sys. (1986), 6, 561-569

Printed in Great Britain

\title{
Multiple attractors in Newton's method
}

\author{
MIKE HURLEY \\ Department of Mathematics and Statistics, Case Western Reserve University, \\ Cleveland, Ohio 44106, USA
}

(Received 29 May 1985)

\begin{abstract}
For each $d \geq 2$ there exists a polynomial $p$ with real coefficients such that the associated Newton function $z-\left[p(z) / p^{\prime}(z)\right]$ has $2 d-2$ distinct attracting periodic orbits in the complex plane. According to a theorem of G. Julia, this is the maximal number of attracting orbits that any rational function of degree $d$ can possess.
\end{abstract}

\section{Introduction}

Perhaps the most common example of iteration of a rational function defined on the complex plane is the use of Newton's method to find the roots of a polynomial. It is widely known that Newton's method may fail to produce a root under certain circumstances. Among the causes of non-convergence, the most robust occurs when the initial point lies in the basin of attraction of an attracting periodic orbit of the Newton function, for this situation is stable under small perturbations of both the initial point and the polynomial. A fundamental problem is to determine if the Newton function of a given polynomial has any such attractors, and if so, how many. If the degree of the polynomial is $d$, then a classical theorem of G. Julia gives an upper bound of $2 d-2$ on the number of attracting periodic orbits (including attracting fixed points) of the Newton function. The main result of this paper is that this upper bound is precise.

THEOREM. If $d \geq 2$, then there is a polynomial of degree $d$ with the property that the Newton function of this polynomial has $2 d-2$ distinct periodic attractors in the complex plane. The polynomial can be chosen to have only real coefficients.

The theorem is proved by making a series of perturbations to a polynomial that has a repeated critical point of high multiplicity. Each perturbation reduces the multiplicity of the degenerate critical point by one and at the same time increases the number of attracting periodic orbits of the associated Newton function by one. The multiplicity of the critical points being dealt with is important; because of it, small changes in the coefficients of the polynomial lead to (locally) large changes in the Newton function. This, combined with the local stability of attracting periodic orbits, enables one to introduce a new attracting orbit without destroying any of the periodic attractors that had been created at previous stages in the construction. 
2. Basic facts about Newton functions

If $p(z)$ is a polynomial over the complex numbers, then the Newton function of $p$, $N p(z)$, is defined by

$$
N p(z)=z-\left[p(z) / p^{\prime}(z)\right] \quad\left(p^{\prime}(z) \neq 0\right) .
$$

The derivative of $N p$ is $p p^{\prime \prime} /\left[p^{\prime}\right]^{2}$. Thus, if the degree of $p$ is $d$, then $N p$ is a rational function with $2 d-2$ critical points. A periodic orbit

is attracting if

$$
\gamma=\left\{z_{0}, N p\left(z_{0}\right),(N p)^{2}\left(z_{0}\right), \ldots,(N p)^{k}\left(z_{0}\right)=z_{0}\right\}
$$

$$
\left|\frac{d}{d z}(N p)^{k}\left(z_{0}\right)\right|<1 .
$$

For example, every root of $p$ is an attracting fixed point of $N p$. If $\gamma$ is an attracting periodic orbit, its basin of attraction is an open set and is defined as

$$
\left\{w \mid \operatorname{dist}\left((N p)^{j}(w), \gamma\right) \rightarrow 0 \text { as } j \rightarrow \infty\right\} \text {. }
$$

Basic to the study of attracting orbits in Newton's method for polynomials is the following result of G. Julia $[\mathbf{J}]$, [Bla].

JULIA'S THEOREM. If $F$ is a rational mapping of the complex plane, of degree at least 2 , then the basin of attraction of any periodic attractor of $F$ contains a critical point of $F$. Consequently the number of attracting periodic orbits is no more than the number of critical points.

In the case of Newton's method for a polynomial of degree $d$, this means that there can be at most $2 d-2$ attracting periodic orbits.

In what follows, the polynomials under consideration will have only real coefficients, so the real line will be an invariant set for the corresponding Newton functions. In this situation, define a band for $N p$ to be a connected component of $\mathbb{R}-\{$ critical points of $p\}$. When $p$ is a polynomial, then $N p$ will have unbounded bands. Certain features of the dynamics of $N p$ on an unbounded band will be relevant to the following discussion. We summarize these features in the following simple lemma, whose proof is an easy calculation. See $[\mathbf{H M}],[\mathbf{H}]$ for more details.

LEMMA 1. Suppose $p$ is a real polynomial of degree $d$.

(a) $(N p)^{\prime}(x) \rightarrow(d-1) / d$ as $x \rightarrow \infty$.

(b) Suppose that $B$ is an unbounded band for $N p$ whose closure contains no roots of $p$. Then $N p(B)=\mathbb{R}$. If $B \neq \mathbb{R}$ then under iteration by $N p$ any point of $B$ will eventually leave $B$.

More complete descriptions of the dynamics of Newton functions on the real line can be found in a number of recent papers, including [SaU], [CoM], [W], [HM], $[\mathbf{H}]$, as well as in the original papers of Barna [B1]-[B4]. A numerical study of Newton functions on the complex plane is contained in [CuGS].

\section{Statement of results}

THEOREM. Given any positive integer $d, d \geq 2$, there is a polynomial $p$ with real coefficients and degree $d$ such that $N p$ has $2 d-2$ distinct periodic attractors in the complex plane. 
In giving a proof of the theorem we will consider the set of polynomials $g$ satisfying the following properties:

(3.1) All of the coefficients of $g$ are real.

(3.2) $g$ has no repeated roots.

(3.3) All of the roots of $g^{\prime}$ are real and lie in $[0, \infty) ; 0$ is a repeated root of $g^{\prime}$, but none of the other roots of $g^{\prime}$ is repeated.

Let $\mathscr{S}$ denote the set of all polynomials satisfying (3.1)-(3.3). The theorem will follow from the following two propositions. In each of them assume that $p$ is a polynomial of the form

(3.4) $p(z)=a_{0}+\sum_{k+1}^{d} a_{j} z^{j}$, with $a_{0}>0, a_{d} \neq 0$, and $k \geq 2$.

Proposition 1. Suppose p satisfies (3.1)-(3.4) and that

(1a) $k$ is odd

(1b) $a_{k+1}>0$

(1c) $p(x)>0$ for all $x \geq 0$;

(1d) $N p$ is monotonic on each of its unbounded bands.

Let $\delta>0$ be given. Then there is a constant $a_{k} \in(-\delta, 0)$ such that if $f(z)=p(z)+a_{k} z^{k}$, then $N f$ has one more attracting periodic orbit than $N p$, and $f$ has the following properties:

(1(i)) $f \in \mathscr{S}$;

(1(ii)) $f(x)>0$ for all $x \geq 0$;

(1(iii)) $f$ has the same number of real roots as $p$;

(1(iv)) Nf has two unbounded bands, and is monotonic on each.

Proposition 2. Suppose p satisfies (3.1)-(3.4) and that

(2a) $k$ is even;

(2b) $a_{k+1}<0$;

(2c) $p(x)>0$ for all $x \leq 0$;

(2d) $N p$ is monotonic on each of its unbounded bands.

Let $\delta>0$ be given. Then there is a constant $a_{k} \in(0, \delta)$ such that if $f(z)=p(z)+a_{k} z^{k}$, then $N f$ has one more attracting periodic orbit than $N p$, and $f$ has the following properties:

(2(i)) $f \in \mathscr{P}$;

(2(ii)) $f(x)>0$ for all $x \leq 0$;

(2(iii)) $f$ has the same number of real roots as $p$;

(2(iv)) Nf has two unbounded bands, and is monotonic on each.

Proof of the theorem in the case $d$ is even. Use the two propositions inductively; in order to satisfy both of the assumptions (1c) and (2c) it is necessary that the degree of $p$ be even. The case $d=2$ is trivial, so assume that $d$ is at least 4 . Define $p_{0}(z)=z^{d}+1$ and note that $N p_{0}$ has $d$ distinct attracting fixed points, none of which is real. Let $p_{1}(z)=f(z)$ where $f$ is obtained by using proposition 1 with $p=p_{0} . N p_{1}$ has $d+1$ distinct periodic attractors and no real roots. Now let $p_{2}=f$ where $f$ is obtained by using proposition 2 with $p=p_{1}$, so $N p_{2}$ has $d+2$ distinct periodic attractors and no real roots. Repeating this process, we eventually obtain $p_{d-2}$ such that $N p_{d-2}$ has $2 d-2$ distinct periodic attractors. 
Remark. The polynomial constructed in this proof satisfies the properties (3.1)-(3.3) and has no real roots.

\section{Proof of propositions 1 and 2}

The proof of the following lemma is straightforward.

LeMma 2. Suppose $p(z)$ is a polynomial and that $L$ is a compact subset of the complex plane that is disjoint from the set of zeros of $p^{\prime}$. Let $f_{\alpha}(z)=p(z)+\alpha z^{k}$. Then $N f_{\alpha}$ converges $C^{1}$ uniformly on $L$ to $N p$ as $\alpha$ tends to 0 .

Proof of proposition 1. For $p$ as in the statement of the proposition let $f_{\alpha}(z)=$ $p(z)+\alpha z^{k}, \alpha$ in $\mathbb{R}$. For $\alpha$ close to 0 the roots of $f_{\alpha}, f_{\alpha}^{\prime}$, and $f_{\alpha}^{\prime \prime}$ will be close to those of $p, p^{\prime}$, and $p^{\prime \prime}$ respectively, so (1(ii)-(iv)) will hold. By lemma 2 and the persistence of periodic attractors under local $C^{1}$ perturbations, for $\alpha$ sufficiently close to $0, N f_{\alpha}$ will have an attracting periodic orbit corresponding to each periodic attractor of $N p$, and of the same period. Additionally, if $\alpha$ is not only close to zero but is also negative, then the assumption that $p$ is in $\mathscr{S}$ will ensure that $f_{\alpha}$ is in $\mathscr{S}$ as well: that (3.1) and (3.2) will be satisfied is obvious; to see that (3.3) is also satisfied we argue as follows. Note that

$$
f_{\alpha}^{\prime}(z)=z^{k-1}\left(k \alpha+\sum_{1}^{d-k} b_{j} z^{j}\right) \quad \text { where } b_{j}=(j+k)\left(a_{j+k}\right),
$$

so 0 is a root of $f_{\alpha}^{\prime}$ of multiplicity $k-1$. Each of the remaining $d-k$ roots of $f_{\alpha}^{\prime}$ has multiplicity 1 ; as $\alpha$ increases to 0 one of these roots converges to 0 and the others converge to the non-zero roots of $p^{\prime}$. The assumptions that $\alpha<0$ and $b_{1}>0$ ensure that for $\alpha$ close enough to 0 all of the roots of $f_{\alpha}^{\prime}$ are real and non-negative. Thus for such $\alpha f_{\alpha}$ will belong to $\mathscr{S}$.

All that remains to show is that $\alpha$ can be chosen so that $N f_{\alpha}$ has an attracting periodic orbit not corresponding to any of the periodic attractors of $N p$. Let $\delta_{0}>0$ be less than $\delta$ and small enough that the preceding arguments hold. For the remainder of the proof consider only values of $\alpha$ satisfying $-\delta_{0}<\alpha<0$. Let $x_{\alpha}$ denote the smallest positive root of $f_{\alpha}^{\prime}$. The argument in the preceding paragraph shows that $x_{\alpha}$ tends to 0 as $\alpha$ increases to 0 . The restriction of $N f_{\alpha}$ to $\mathbb{R}$ has vertical asymptotes at 0 and at $x_{\alpha}$. Between these asymptotes $f_{\alpha}$ is positive and $f_{\alpha}^{\prime}$ is negative, so that $N f_{\alpha}(x)>x$. Consequently $N f_{\alpha}$ is bounded below on $\left(0, x_{\alpha}\right)$ and has a turning point inside this interval. In fact, it is easy to check that

$$
\inf \left\{N f_{\alpha}(x) \mid 0<x<x_{\alpha}\right\} \rightarrow \infty
$$

as $\alpha$ tends to 0 from below. Let $y_{\alpha}$ denote the smallest positive critical point of $N f_{\alpha}$. By (1(ii)) and the fact that $\left(N f_{\alpha}\right)^{\prime}=f_{\alpha} f_{\alpha}^{\prime \prime} /\left(f_{\alpha}^{\prime}\right)^{2}$ it follows that $y_{\alpha}$ is the least positive root of $f_{\alpha}^{\prime \prime}$. Since $f_{\alpha}^{\prime \prime}$ is given by

$$
z^{k-2}\left(\alpha k(k-1)+\sum_{1}^{d-k} c_{j} z^{j}\right) \quad \text { with } c_{j}=(j+k)(j+k-1) a_{j+k}
$$

and $c_{1}>0$, the map $\alpha \rightarrow y_{\alpha}$ is smooth for $-\delta_{1} \leq \alpha<0$ for some $\delta_{1}$ chosen to satisfy $0<\delta_{1}<\delta_{0}$, and $y_{\alpha} \rightarrow 0$ as $\alpha$ increases to 0 . 
Claim. There are values of $\alpha$ in $\left(-\delta_{1}, 0\right)$ for which $y_{\alpha}$ lies on an attracting periodic orbit of $N f_{\alpha}$.

Since $\left(N f_{\alpha}\right)^{\prime}\left(y_{\alpha}\right)=0$, a periodic orbit containing $y_{\alpha}$ is necessarily attracting.

Proof of claim. Let $q_{\alpha}$ denote the largest real root of $f_{\alpha}^{\prime}$ (so $q_{\alpha} \geq x_{\alpha}>y_{\alpha}>0$ ). The graph of $N f_{\alpha}$ for $x$ near 0 is depicted in figure 1. By (1(iv)) and lemma $1, N f_{\alpha}$ is

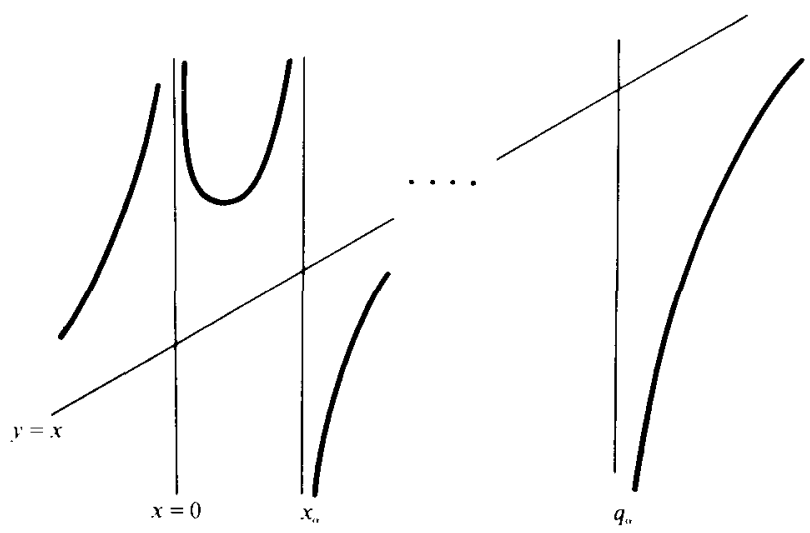

FIGURE 1

monotonic on $\left(q_{\alpha}, \infty\right)$ and maps $\left(q_{\alpha}, \infty\right)$ onto the real line. Thus there is a unique inyerse image of $y_{\alpha}$ under $N f_{\alpha}$ that lies in $\left(q_{\alpha}, \infty\right)$. We will show that for certain values of $\alpha$ this pre-image of $y_{\alpha}$ is also a forward iterate of $y_{\alpha}$. Note that as $\alpha$ approaches 0 from below the number of consecutive iterates of $N f_{\alpha}\left(y_{\alpha}\right)$ that are greater than $q_{\alpha}$ tends to infinity. This holds because $N f_{\alpha}\left(y_{\alpha}\right) \rightarrow \infty$ as $\alpha$ increases to 0 (by (4.1)) and $\left|N f_{\alpha}(x)-(d-1) x / d\right|$ has a constant limit as $x \rightarrow \infty$ (and these limits are uniform for $\alpha$ in a compact set). Choose $\alpha_{0}$ in $\left(-\delta_{1}, 0\right)$ and $m>1$ so that for $\alpha=\alpha_{0}, N f_{\alpha}\left(y_{\alpha}\right)>q_{\alpha}$ and $\left(N f_{\alpha}\right)^{m}\left(y_{\alpha}\right)<q_{\alpha}$. Define

$$
\alpha(m)=\inf \left\{\beta<0 \mid\left(N f_{\alpha}\right)^{j}\left(y_{\alpha}\right)>q_{\alpha} \text { for all } 1 \leq j \leq m \text { and } \beta<\alpha<0\right\} .
$$

For some $j(m) \leq m$ we have $N f_{\alpha(m)}^{j(m)}\left(y_{\alpha(m)}\right)=q_{\alpha(m)}$ and $N f_{\alpha}^{j(m)}\left(y_{\alpha}\right)>q_{\alpha}$ for $\alpha(m)<\alpha<0$. Let $T=j(m)+1$. The construction ensures that the map $G(\alpha)=\left(N f_{\alpha}\right)^{T}\left(y_{\alpha}\right)$ is continuous on $(\alpha(m), 0)$, and

$$
\begin{aligned}
& G(\alpha) \rightarrow-\infty \quad \text { as } \alpha \text { decreases to } \alpha(m) \\
& G(\alpha) \rightarrow \infty \quad \text { as } \alpha \text { increases to } 0 .
\end{aligned}
$$

Since the curve $\alpha \rightarrow y_{\alpha}$ is continuous and bounded on $\left(-\delta_{1}, 0\right)$, this curve and the graph of $G(\alpha)$ must intersect (see figure 2). At a point of intersection we have $\left(N f_{\alpha}\right)^{T}\left(y_{\alpha}\right)=y_{\alpha}$.

The proof of proposition 2 is analogous to that of proposition 1 . In it there is a critical point of $N f_{\alpha}$ that is positive, near 0 , and which is mapped toward $-\infty$ as $\alpha \rightarrow 0$. The graph of $N f_{\alpha}$ in this situation is shown in figure 3 . 


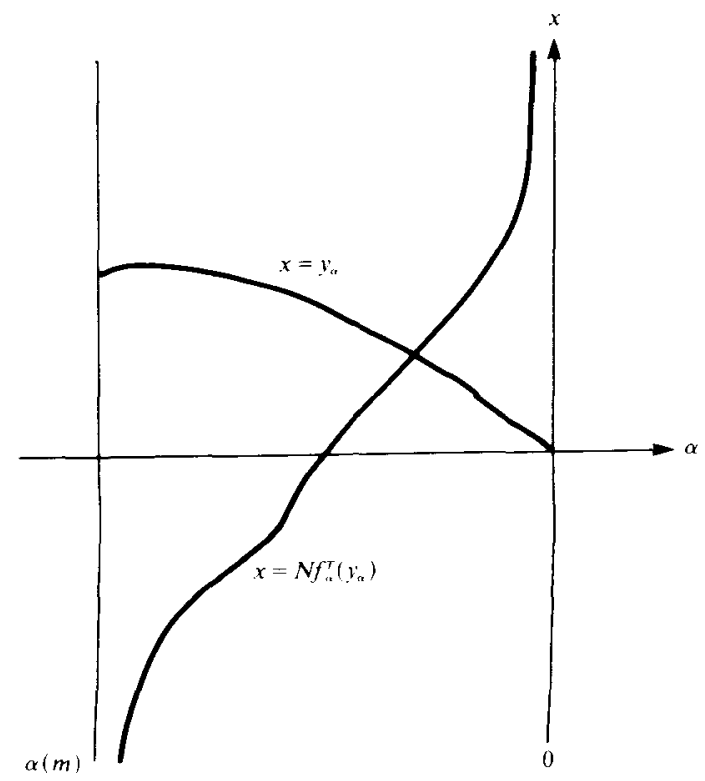

FIGURE 2

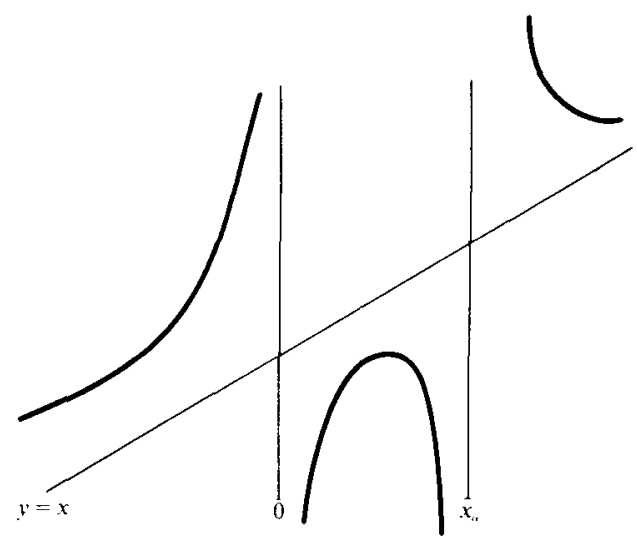

FIGURE 3

\section{Proof of the theorem when $d$ is odd}

Suppose that $d$ is odd and at least 3 . Use the construction of $\S 4$ to obtain a real polynomial $p$ satisfying

(5.1) degree $(p)=d-1$.

(5.2) $p$ satisfies (3.1)-(3.3).

(5.3) $p(x)>0$ for all $x$ in $\mathbb{R}$.

(5.4) $N p$ has $2 d-4$ distinct attracting periodic orbits in the complex plane. Consider polynomials of the form

$$
g_{\alpha}(z)=p(z)+\alpha z^{d}, \quad \alpha>0 .
$$


Each $g_{\alpha}$ has a single real root, which is negative, and this root tends to $-\infty$ as $\alpha$ decreases to 0 . By lemma 2, if $\alpha>0$ is small enough, $N g_{\alpha}$ will have $2 d-3$ distinct attracting periodic orbits: orbits corresponding to each of the periodic attractors of $N p$ as well as an attracting fixed point at the real root of $g_{\alpha}$. To see that there are small positive values of $\alpha$ for which $N g_{\alpha}$ has $2 d-2$ distinct attractors, consider the graph of $\mathrm{Ng}_{\alpha}$ (see figure 4).

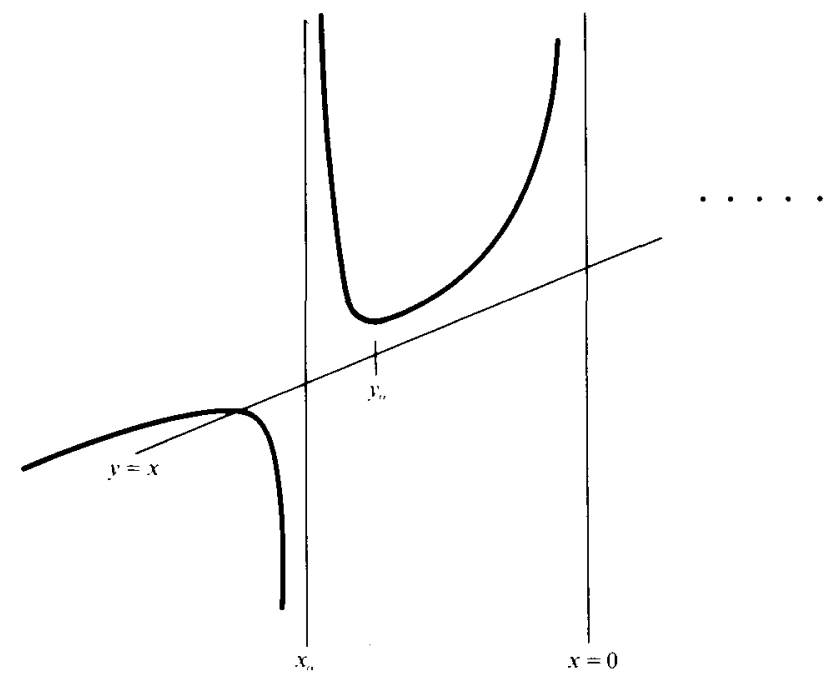

FIGURE 4

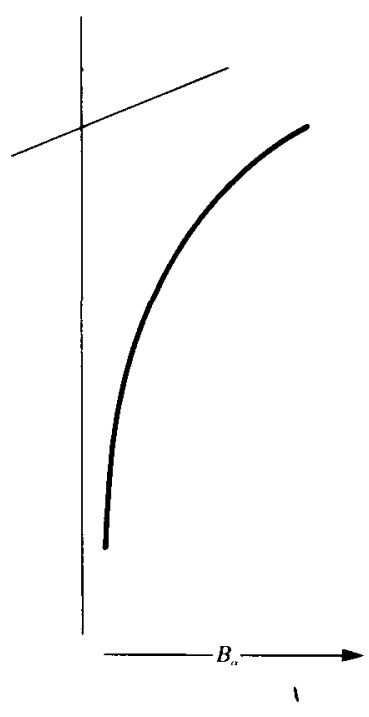

By (3.3) $p^{\prime}$ has no roots on the negative real axis, so using (5.5) it is an easy argument to show that $g_{\alpha}^{\prime}(0)=0$ and that $g_{\alpha}^{\prime}$ has exactly one negative root at a point that we label $x_{\alpha}$. Thus the negative real axis contains two bands for $N g_{\alpha}$, an unbounded one $\left(-\infty, x_{\alpha}\right)$ that contains the negative root of $g_{\alpha}$, and a bounded one $\left(x_{\alpha}, 0\right)$ with the property that $N g_{\alpha}(x)>x$ whenever $x_{\alpha}<x<0 . N g_{\alpha}$ is bounded below on $\left(x_{\alpha}, 0\right)$ and has a minimum on this band, which occurs at a point $y_{\alpha}$ that is a root of $g_{\alpha}^{\prime \prime}$. (For $\alpha$ small the other roots of $g_{\alpha}^{\prime \prime}$ are near those of $p^{\prime \prime}$, so a counting argument shows that $y_{\alpha}$ is the only turning point of $N g_{\alpha}$ in the band $\left(x_{\alpha}, 0\right)$.) Note that both $x_{\alpha}$ and $y_{\alpha}$ tend to $-\infty$ as $\alpha$ decreases to 0 .

Because $g_{\alpha}^{\prime}(0)=0$ there is an unbounded band for $N g_{\alpha}$ that lies in the positive half-line; call this band $B_{\alpha}$. Since the only real root of $g_{\alpha}$ is negative, lemma 1 shows that $N g_{\alpha}$ maps $B_{\alpha}$ onto $\mathbb{R}$. We will show that for certain small values of $\alpha$ the orbit of $y_{\alpha}$ is periodic and is contained in the union of $B_{\alpha}$ and $\left(x_{\alpha}, 0\right)$.

Define

$$
K(\alpha)=\min \left\{j \mid\left(N g_{\alpha}\right)^{j+1}\left(y_{\alpha}\right)>0\right\} .
$$

Claim. $K(\alpha)$ goes to infinity as $\alpha$ decreases to 0 .

This claim follows from the following facts.

(5.6) $N g_{\alpha}$ has its minimum on $\left(x_{\alpha}, 0\right)$ at $y_{\alpha}$.

(5.7) $N g_{\alpha}$ converges uniformly to $N p$ as $\alpha$ decreases to 0 on compact subsets of the negative real axis. 
(5.8) $N p(x)$ tends to $-\infty$ as $x \rightarrow-\infty$.

(5.9) $|N p(x)-[(d-1) /(d-2)] x|$ has a finite limit as $x \rightarrow-\infty$.

Now choose $\delta>0$ small enough that if $0<\alpha<\delta$, then $N g_{\alpha}$ has the features described above. Pick some $\alpha_{1}$ in $(0, \delta)$ and let $m_{0}$ be a fixed integer greater than $K\left(\alpha_{1}\right)$. Define

$$
\alpha_{0}=\inf \left\{0<\alpha<\delta \mid K(\alpha) \leq m_{0}\right\} .
$$

For any $j$ satisfying $0 \leq j \leq m_{0}+1$, the map $\alpha \rightarrow\left(N g_{\alpha}^{j}\right)\left(y_{\alpha}\right)$ is continuous on $0<\alpha$ $<\alpha_{0}$, and there is an integer $m \leq m_{0}$ such that $\left(N g_{\alpha}\right)^{m}\left(y_{\alpha}\right)$ tends to 0 from below as $\alpha$ tends to $\alpha_{0}$ from below. Consequently, $\left(N g_{\alpha}\right)^{m+1}\left(y_{\alpha}\right)$ goes to infinity as $\alpha \rightarrow \alpha_{0}$ from below. On the other hand, (5.6)-(5.9) combine to show that $\left(N g_{\alpha}\right)^{m+1}\left(y_{\alpha}\right)$ goes to $-\infty$ as $\alpha$ tends to 0 from above.

Now consider inverse images of $y_{\alpha}$. For each small positive $\alpha$ there is a point $r_{\alpha}$ in $B_{\alpha}$ defined by $N g_{\alpha}\left(r_{\alpha}\right)=y_{\alpha}$. It is not hard to check that for $0<\alpha<\alpha_{0}$ the function $\alpha \rightarrow r_{\alpha}$ is continuous (and nearly constant if $\alpha_{0}$ is small). One concludes that if the two curves $\alpha \rightarrow r_{\alpha}$ and $\alpha \rightarrow\left(N g_{\alpha}\right)^{m+1}\left(y_{\alpha}\right)$ are graphed in the $(\alpha, x)$-plane, then the two graphs must intersect, as shown in figure 5. If $(\alpha, x)=(A, X)$ is the point of intersection, then

$$
\left(N g_{A}\right)^{m+2}\left(y_{A}\right)=N g_{A}\left(\left(N g_{A}\right)^{m+1}\left(y_{A}\right)\right)=N g_{A}(X)=y_{A}
$$

Thus $N g_{A}$ is a rational function of degree $d$ with $2 d-2$ distinct attracting periodic orbits.

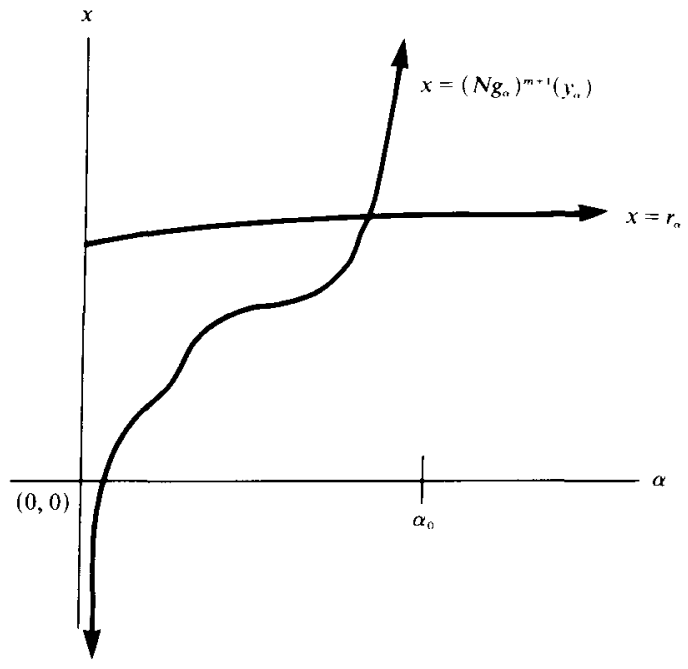

FIGURE 5

\section{REFERENCES}

[B1] B. Barna. Uber das Newtonsche Verfahren zur Annaherung von wurzeln algebraischen Gleichungen. Publ. Math. Debrecen. 2 (1951), 50-63.

[B2-4] B. Barna. Uber die Divergenzpunkte des Newtonschen Verfahrens zur bestimmung von wurzeln algebraischen Gleichungen:

I. Publ. Math. Debrecen, 3 (1953), 109-118.

II. Publ. Math. Debrecen, 4 (1956), 384-397.

III. Publ. Math. Debrecen, 8 (1961), 193, 207. 
[Bla] P. Blanchard. Complex analytic dynamics on the Riemann sphere. Bull. Amer. Math. Soc. 11 (1984), 85-141.

[CoM] M. Cosnard \& C. Masse. Convergence presque partout de la méthode de Newton. C.R. Acad. Sci. Paris 297 (1983), 549-552.

[CuGS] J. Curry, L. Garnett \& D. Sullivan. On the iteration of a rational function: computer experiments with Newton's method. Comm. Math. Phys. 91 (1983), 267-277.

[H] M. Hurley. Attracting orbits in Newton's method. Trans. Amer. Math. Soc. To appear.

[HM] M. Hurley \& C. Martin. Newton's algorithm and chaotic dynamical systems. SIAM I. Math. Anal. 15 (1984), 238-252.

[J] G. Julia. Memoire sur l'iteration des fonctions rationnelles. J. Math. 8 (1918), 47-245.

[SaU] D. Saari \& J. Urenko. Newton's method, circle maps, and chaotic motion. Amer. Math. Monthly 91 (1985), 3-17.

[Sm] S. Smale. The fundamental theorem of algebra and complexity theory. Bull. Amer. Math. Soc. 4 (1981), 1-36.

[Sul] D. Sullivan. Conformal dynamical systems. pp. 725-752 in Geometric Dynamics, J. Palis edit., (Springer Lect. Notes in Math. 1007). Springer-Verlag, New York, 1983.

[W] S. Wong. Newton's Method and symbolic dynamics. Proc. Amer. Math. Soc. 91 (1984), $245-253$. See also S. Wong. The instability of the period two cycles of Newton's Method, preprint. 